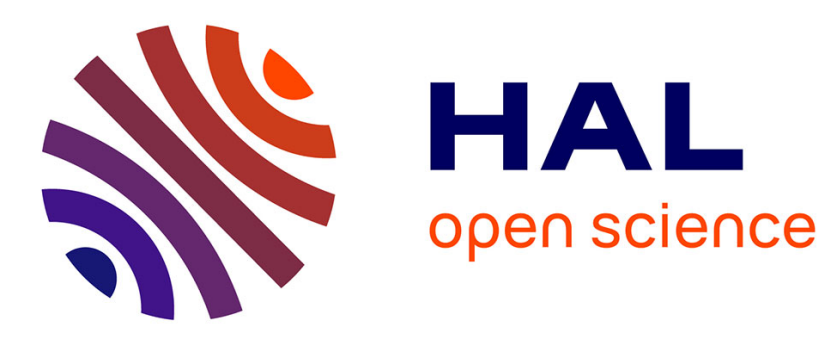

\title{
Leidenfrost levitated liquid tori
}

Stéphane Perrard, Yves Couder, Emmanuel Fort, Laurent Limat

\section{To cite this version:}

Stéphane Perrard, Yves Couder, Emmanuel Fort, Laurent Limat. Leidenfrost levitated liquid tori. EPL - Europhysics Letters, 2012, 100 (5), pp.54006. 10.1209/0295-5075/100/54006 . hal-02358402

\section{HAL Id: hal-02358402 \\ https://hal.science/hal-02358402}

Submitted on 14 Nov 2019

HAL is a multi-disciplinary open access archive for the deposit and dissemination of scientific research documents, whether they are published or not. The documents may come from teaching and research institutions in France or abroad, or from public or private research centers.
L'archive ouverte pluridisciplinaire HAL, est destinée au dépôt et à la diffusion de documents scientifiques de niveau recherche, publiés ou non, émanant des établissements d'enseignement et de recherche français ou étrangers, des laboratoires publics ou privés. 


\title{
Leidenfrost levitated liquid tori
}

\author{
S. Perrard, ${ }^{1}$ Y. Couder, ${ }^{1}$ E. Fort ${ }^{2}$ and L. Limat ${ }^{1}$ \\ ${ }^{1}$ Matière et Systèmes Complexes, CNRS and Université Paris Diderot UMR 7057, \\ Bâtiment Condorcet, 10 rue Alice Domon et Léonie Duquet, 75013 Paris, France \\ ${ }^{2}$ Institut Langevin, CNRS, ESPCI ParisTech and Université Paris \\ Diderot, UMR 7587, 1 rue Jussieu, 75238 Paris Cedex 05, France
}

\begin{abstract}
Levitating a liquid over a vapor film was limited to droplets. Here we show that on curved substrates a larger quantity of fluid can be suspended. This opens a new possibility for exploring new free liquid surface phenomena without any contact with a solid. In one of the simplest possible situation, a large fluid torus is levitated over a circular trough. A poloidal flow inside the ring generates a wave on its inner side, making it polygonal. This wave is described by a solitonic model which balances surface tension and a pressure depletion due to the distortion of the poloidal flow.
\end{abstract}

PACS numbers: 47 55.D-, Drops and bubbles, 47.85.mf Lubrication flows, 47.55.pf Marangoni convection, 47.20.Ma Interfacial instabilities , 47.35.Pq, Capillary waves

The situations in which any fluid can be steadily kept isolated from any contact with a dense medium (solid or liquid) are scarce and often obtained in extreme situations like microgravity for liquids or electromagnetic confinement for toroidal plasmas. A third (and more accessible) possibility is provided by the Leidenfrost effect [1] where a liquid, deposited on a very hot plate, is kept levitating over the thin vapor film created beneath the drop by evaporation. On a planar plate this possibility is, however, limited to drops having a maximum size of approximately twice the capillary length $l_{c}=\sqrt{\gamma / \rho g}$ (where $\gamma$ is the surface tension, $\rho_{f}$ the fluid density and $g$ gravity). For these drops of a diameter on the order of one centimeter (for water) the capillary and gravity effects are comparable. That is the Laplace pressure high enough to limit the deformation of the drop by the vapor under it, so that the vapor can easily escape by the side of the near contact area[2,3]. If a larger quantity of liquid is deposited, the effect of gravity causes the drop to take a pancake shape of thickness of $2 l_{c}$. The region of near contact becomes too large, and all the vapor generated underneath cannot entirely escape around the edges. Some of it accumulates and forms bubbles that move through the fluid layer and disturb it violently [4]. Because of this limitation in size, even though it provides a free surface situation, the use of Leidenfrost effect was limited to the investigation of capillarity dominated effects, such as the deformation modes of a drop[5], or self propulsion on a ratchet substrate [6].

The aim of the present article is two-fold. We first show that by the use of non-planar substrates it is possible to levitate quantities of liquid orders of magnitude larger than the traditional Leidenfrost effect. As an example we show in a second part that the waves propagating on one type of levitated liquid rings are generated by a secondary instability of the toroidal vortex it contains. We then illustrate that obtaining a fluid bounded only by free surfaces is a tool for the investigation of the coupling between hydrodynamics instabilities and surface waves.

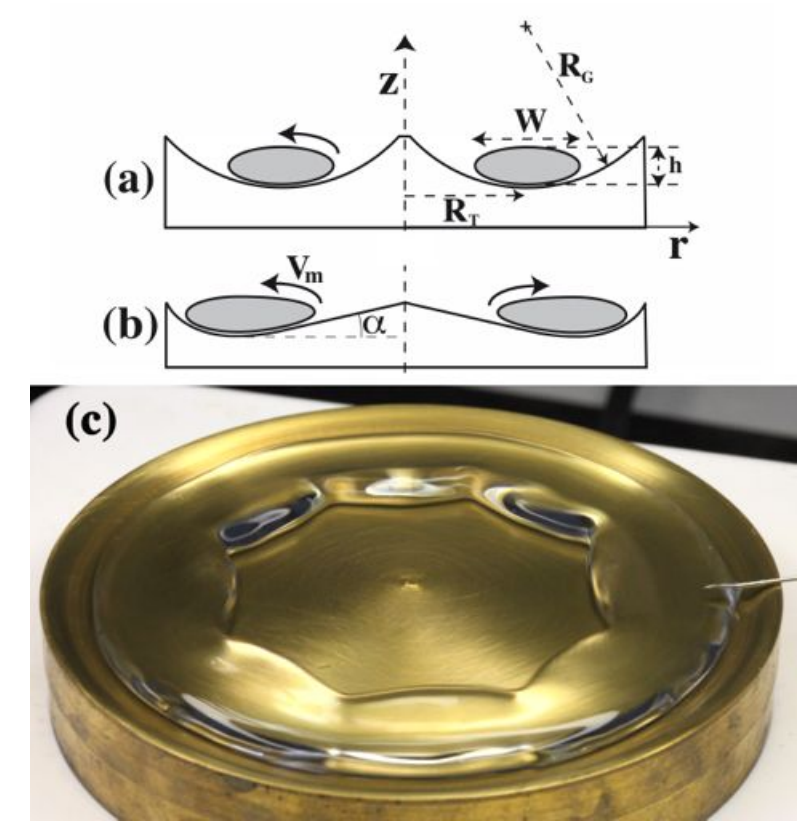

FIG. 1: (a) Sketch of the toroidal configuration. (b) An asymmetric variant with a trough having a gentle slope inward and a steep slope outward. (c) Photograph of a polygonal pattern observed in the configuration (b).

In order to circumvent the limitation of size, the idea is to use non-planar substrates where one of the main curvatures is large everywhere so that the water vapor can always escape easily in one direction. A large number of possible configurations of troughs meet these requirements. They can be linear, circular or even form various grid patterns. In this letter, we limit ourselves to a simple geometry where the substrate has only one circular trough, which allows us to stabilize a large curved levitating liquid cylinder with a periodic boundary condition: a ring (see Fig. 1).

The substrates we used are $20 \mathrm{~mm}$ thick brass plates with circular troughs of several profiles and different radii $R_{G}$ 
(see fig.1). The brass substrate is placed on a horizontal $1000 \mathrm{~W}$ temperature-regulated hot plate heated at $T_{S}$ between $200^{\circ} \mathrm{C}$ and $400^{\circ} \mathrm{C}$. When distilled water is poured into the trough, a large crescent-shaped levitated drop forms. When more water is added the extremities of the crescent drop connect: a levitated liquid torus is formed. It thins down with time due to evaporation. In order to compensate this natural decay, we used a motorized syringe to create a weak constant flow $Q$ of hot water into the levitating annulus. The torus thus eventually reaches a steady regime, where evaporation and injection rates are equal.

Only the surface of the transverse section is now limited: by the appearance of bubbles since the width and by the capillary length for the height. These limitations lead to a maximum area of the vertical section of few $\mathrm{cm}^{2}$. However we haven't observed any limit in the azimuthal direction. A liter of water should be set in levitation with a circular trough of radius $30 \mathrm{~cm}$. The profile of the section of the liquid torus depends on the substrate geometry defined in Fig. 1. If the trough is deep and narrow (i.e. with $r_{G}$ of the order of few $l_{c}$ and $r_{G}<R_{T}$ ), a stable liquid annulus is obtained. On shallower troughs as shown in Fig. 1(b) where the radius $r_{G}>R_{T}$ the levitated liquid torus is flattened by gravity into a doughnut shape and an interesting phenomenon is observed at the surface on which we will now focus.

We study steady regimes that are controlled by the temperature of the substrate and the injection rate. At low substrate temperature $\left(T<220^{\circ} \mathrm{C}\right)$ and when the annulus has a small section the levitated liquid is at rest and a stable annulus is observed. As the temperature is increased there is a threshold depending both on temperature and torus dimension above which a poloidal flow appears, the fluid rises up on the inner side of the ring. These fluid motions are sketched in Fig. 1(b). Such motions inside Leidenfrost drops can be driven either by surface tension gradient [5] or by the escape motion of the vapor layer $[6,7]$, or by both. The study of this threshold is beyond our present aim. The local velocities at the surface of the torus have been measured by following the motion of tracers of diameter $d=50 \mu \mathrm{m}$ as they are trapped by surface tension at the surface. The mean velocity $V_{m}$ is of the order of $50 \mathrm{~mm} / \mathrm{s}$ and does not depend on the torus width $W$.

The striking observation is that when this liquid flow appears, a propagative wave grows on the internal side of the torus, forming a polygonal pattern rotating in the laboratory frame (see Fig. 2). On all the shallow substrates of the type shown in Figs. 1(a) and 1(b), the permanent injection of water leads to stable and reproducible polygonal patterns (see Figs. 1(c) and 2).

In order to study this instability quantitatively, we used specially designed substrates in which the trough has an asymmetrical profile (see Fig. 1(b)) with a weak constant slope inwards and a steep edge outwards that fixes the

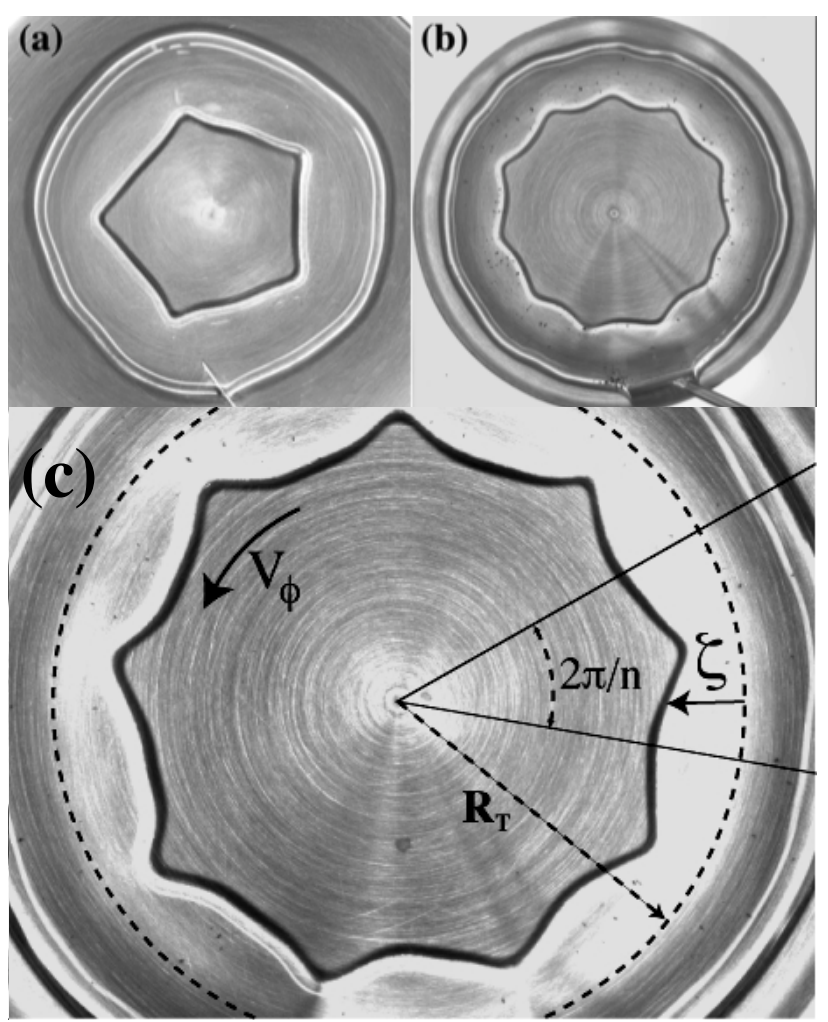

FIG. 2: Photographs of three polygonal modes observed on two different substrates: (a) for $R_{T}=30 \mathrm{~mm}$, (b) and (c) for $R_{T}=50 \mathrm{~mm}$, using two different volume of water, which correspond to different number of sides.

external perimeter of the doughnut. The number $n$ of sides increases continuously with the donut's perimeter. Using three substrates of different perimeters $2 \pi R_{T}$, we observed polygons having from three to twelve sides (see Fig. 2). The wavelength $\lambda=2 \pi R_{T} / n$ is found to be of the order of the width $W$ so that $n \propto R_{T} / W$ (see Fig. 3). In the laboratory frame, these polygonal patterns rotate along the azimuthal direction $\theta$ with a linear velocity $V_{\theta}$. The rotation has an equal probability to be clockwise or counterclockwise, but this degeneracy can be lifted. Since the friction of the liquid on the substrate is very weak, the whole liquid annulus can be set into an azimuthal rotation with a velocity $U$ by means of a weak air jet impinging tangentially on the upper surface. The wave is then always counter-propagating so as to minimize its velocity in the laboratory frame. For all values of $U$, the velocity $V_{\theta}$ of the wave is constant in the liquid frame of reference. We find $V_{\theta}=105 \pm 5 \mathrm{~mm} / \mathrm{s}$ for the asymmetrical trough shown in Fig. 1(b). This is two times lower than the usual minimum of velocity for capillary-gravity waves at an equivalent depth of $5 \mathrm{~mm}$ corresponding to the height of the levitated ring. It can be however well understood by taking into account the fact that the interface does not move vertically but instead along the substrate slope forming an angle $\alpha=5^{\circ}$ with the hor- 


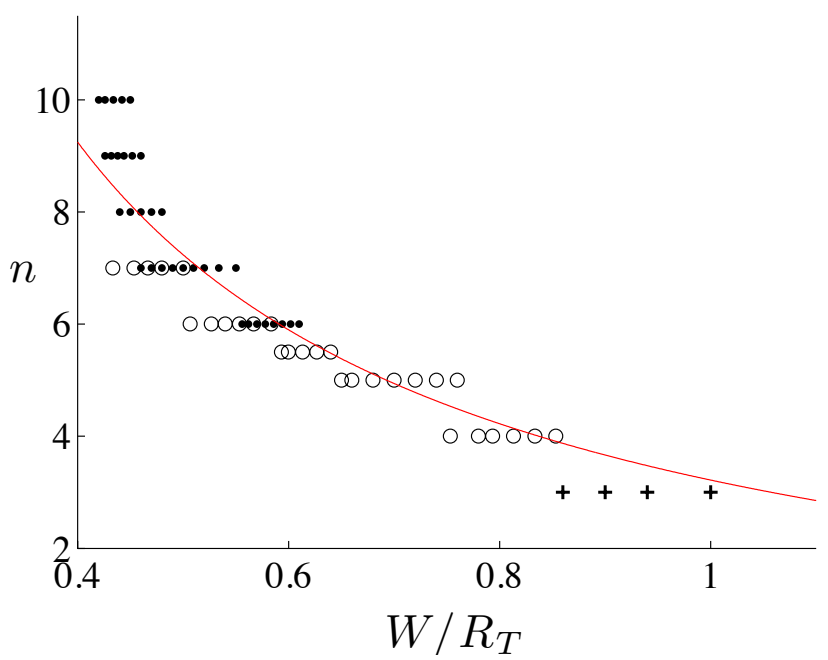

FIG. 3: Number of polygon sides as a function of the aspect ratio $W / R_{T}$ of the torus for three different substrates: $R_{T}=$ $15 \mathrm{~mm}(+), R_{T}=30 \mathrm{~mm}(\circ)$ and $R_{T}=50 \mathrm{~mm} \mathrm{( \cdot )}$. The wavelength $\lambda=2 \pi R_{T} / n$ is approximately proportional to the torus thickness so that $n \sim R_{T} / W$ (line).

izontal. A good interpretation of the observed velocity is obtained by replacing the usual dispersion relation of surface waves $g$ with a reduced value $g_{r}=g \sin \alpha$ and the fluid depth by the width $W$ of the torus.

The shape of the polygons, the experimental geometry, and the liquid flow inside the torus are reminiscent of other faceting instabilities observed in partially filled rotating tanks [8, 9], and in circular hydraulic jumps $[10,11]$. In all these systems, a circular front limiting the boundaries of a toroidal vortex becomes polygonal. This constitutes one of the most intriguing phenomena observed at the frontier between vortex dynamics and free surface flows. An open question is whether the mechanism of instability is the same in all these systems $[12,13]$. Our experimental set-up offers the opportunity to study this question in ideal conditions, i.e. where the vortex is well delimited as it coincides with the position of the free surface.

When zooming in one wavelength, an obvious feature appears: the strong asymmetry between the regions where the fluid protrudes inwards (the sides of the polygons) and the constrictions (the corners). The position of the inner side interface is defined by $\zeta(\theta)$ the radial distance to the azimuthal axis $R_{T}$ of the torus using the conventions given in Fig. 2. For a given mode the amplitude of the wave increases with the imposed temperature. We measured systematically the wave profile $A(\theta)=\zeta-\zeta_{\max }$ for various amplitudes $A_{0}=\left|\zeta_{\min }-\zeta_{\max }\right|$. They are shown in Fig. 4(a) for a pattern $n=6$. In order to characterize these shapes we measured the angular width $\Delta \theta$ at mid-height as a function of the amplitude $A_{0}$ for all patterns (see Fig. 4(b)). The patterns of various orders can thus be compared. Figure 4(c) is a plot of $\zeta$ as a function
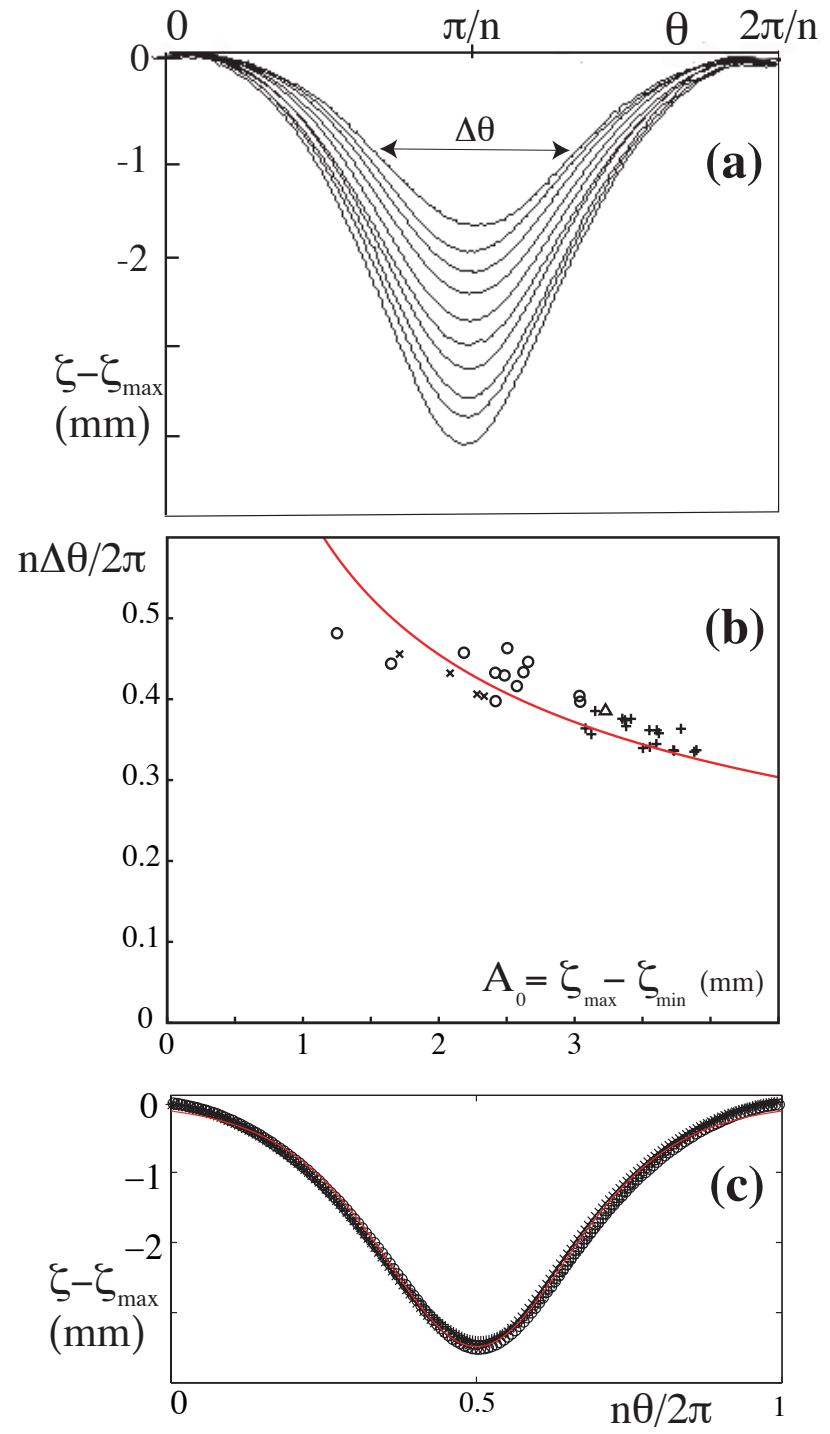

FIG. 4: (a) The wave profiles $\zeta$ of increasing amplitude due to a substrate temperature between $220^{\circ} \mathrm{C}$ and $400^{\circ} \mathrm{C}$. (b) Evolution of the normalized half-width $n \Delta \theta / 2 \pi$ as a function of the wave amplitude (for $n=5(\triangle), 6(\square), 7(\times), 8(\circ), 9(+)$ ). (c) The superposition of renormalized wave profiles for three different patterns $(\mathrm{n}=6(\square), 7(\times), 8(\circ))$. The continuous line is a fit with $n \Delta \theta / 2 \pi \propto A_{0}^{1 / 2}$ (see eq.4).

of the normalized angle $n \theta / 2 \pi$ for patterns with $n=6,7,8$ where the wave amplitude is the same. The wave profile remains identical, the apparent differences observed in Fig. 2 being only due to the mean curvature imposed at large scale by the geometry. This suggests that we can describe the wave profile by a universal equation with only one parameter fixing both its amplitude and its width. Here we propose a simple heuristic model to obtain such an equation.

A characteristic of a torus is that its inner side has a perimeter shorter than the outer side. For this reason the toroidal geometry induces by flow conservation a 
velocity gradient between the two vertical interfaces at $r=r_{\min }$ and $r=r_{\max }$. This gradient has been measured by using the same tracers as previously. We found $\delta(V)=V\left(r_{\min }\right)-V\left(r_{\max }\right) \sim 60 \mathrm{~mm} / \mathrm{s}$. Applying the Bernoulli relation to the streamline at the free surface of the torus we obtain a pressure difference $\Delta P_{v}$ :

$$
\Delta P_{v}=\frac{1}{2} \rho \delta\left(V^{2}\right)=\frac{1}{2} \rho\left(V^{2}\left(r_{\min }\right)-V^{2}\left(r_{\max }\right)\right) \sim 2 \mathrm{~Pa}
$$

On the other hand, the horizontal curvature of the internal perimeter $\Gamma=1 / r_{\min }$ creates a capillary pressure jump $\Delta P_{\gamma}=\gamma / r_{\text {min }} \sim 2 \mathrm{~Pa}$ which tends to maintain this perimeter circular. Note that these two differences of pressure at the interface are much smaller than both the hydrostatic pressure and the Laplace pressure coming from the vertical curvature of the interface. These main terms lead to the propagation of the waves when a deformation appears. However, we have observed the spontaneous growth of waves only when a poloidal flow exists inside the torus. Thus we assume that an increase of Bernoulli pressure is induced by the reorganization of the flow near the corner, and pulls the interface towards the liquid phase. We assume the simplest possible expression for this Bernouilli extra pressure, i.e. $\delta\left(V^{2}\right) / V^{2} \sim-\beta_{1} A / h+\beta_{2} A^{2}$ where $\beta_{1}$ and $\beta_{2}$ are constants. The interface deformation can be limited by two different forces. An increase of the capillary pressure tends to limit the distortions of the internal torus perimeter. On the other hand the modification of the hydrostatic pressure $\rho g \sin \alpha\left(\zeta-\zeta_{\max }\right)$, as the interface moves along the slope of the substrate, could limit the amplitude. Roughly, we can admit that the wave profile of the internal perimeter is ruled by the following pressure equilibrium:

$$
\frac{\rho}{2} \delta\left(V^{2}\right)=\frac{\gamma}{r_{\min }} \frac{\partial^{2} \zeta}{\partial \theta^{2}}-\rho g \sin \alpha\left(\zeta-\zeta_{\max }\right)
$$

Defining an amplitude as $A=\zeta-\zeta_{\max }$ and using the previous expression suggested for $\delta\left(V^{2}\right)$ one arrives at an equation that reads:

$$
\frac{\partial^{2} A}{\partial \theta^{2}}=-\beta_{1}\left(W e-W e_{c}\right) A+\beta_{2} A^{2}
$$

in which $W e$ is a Weber number defined as $W e=$ $\rho r_{\text {min }} V^{2} / \gamma$. We $e_{c}$ is a critical value which depend on the reduced gravity term $g \sin \alpha$, and $\beta_{2} A^{2}$ is a nonlinear term of second order that we can expect to hold because of the lack of symmetry between $-A$ and $+A$. Finally the gravity term is responsible for the threshold, and the wave profile is a local equilibrium between a dynamical difference of pressure created by the flow and a Laplace pressure. This amplitude equation is analogous to the Korteweg de Vries one, describing a large family of non linear waves [14]. Above a strong enough forcing
$\left(W e>W e_{c}\right)$, our equation admits localized solutions :

$$
A=-A_{0} \operatorname{sech}^{2} \frac{2 \pi \theta}{n \Delta \theta}
$$

As one can verify in fig.4(c), this shape describes the observed profile for our corners, and the scaling $n \Delta \theta /(2 \pi) \sim A_{0}^{-1 / 2}$ implied by Eq.3 holds reasonably well with all the collected data.

A remarkable fact in this experiment is that the amplitude of these solitons is negative. This has never been observed at this wavelength, corresponding usually to gravity waves. The sole observation of a dark soliton in hydrodynamic waves is due to Falcon et al. [15] for a wavelength ten times smaller than ours, in a capillary regime where the amplitude can become negative.

Acknowledgements. We are grateful to J. Gosselin, S. Kosgodagan, J. Moukhtar, A. Eddi, D. Razis, L. Rhea, A. Roger, L. Royon, C. T. Pham, for their help at various stages of this work and to J. Bush and M. Labousse for useful discussions. This work was sponsored by the French National Agency for Research.

[1] J. G. Leidenfrost, De Aquae Communis Nonnullis Qualitatibus Tractatus (Duisburg, 1756).

[2] J. H. Snoeijer, P. Brunet, and J. Eggers, Phys. Rev. E 79 (2009).

[3] L. Duchemin, J. R. Lister, and U. Lange, J. Fluid Mech. 533, 161 (2005).

[4] A. L. Biance, C. Clanet, and D. Quere, Phys. of Fluids 15, 1632 (2003).

[5] N. Tokugawa and R. Takaki, J. Phys. Soc. Japan 63 (1994).

[6] H. Linke, B. J. Aleman, L. D. Melling, M. J. Taormina, M. J. Francis, C. C. Dow-Hygelund, V. Narayanan, R. P. Taylor, and A. Stout, Phys. Rev. Lett. 96 (2006).

[7] A. Snezhko, E. B. Jacob, and I. S. Aranson, New Journal of Physics 10 (2008).

[8] G. H. Vatistas, J. Fluid Mech. 217 (1990).

[9] T. R. N. Jansson, M. P. Haspang, K. H. Jensen, P. Hersen, and T. Bohr, Phys. Rev. Lett. 96 (2006).

[10] C. Ellegaard, A. E. Hansen, A. Haaning, A. Marcussen, T. Bohr, J. Hansen, and S. Watanabe, Nature 392, 767 (1998).

[11] J. W. M. Bush, J. M. Aristoff, and A. E. Hosoi, J. Fluid Mech. 558, 33 (2006).

[12] C. Ellegaard, A. E. Hansen, A. Haaning, A. Marcussen, T. Bohr, J. Hansen, and S. Watanabe, Nonlinearity 12, 1 (1999).

[13] R. Bergman, L. Tophoj, T. Homan, P. Hersen, A. Andersen, and T. Bohr, J. Fluid Mech. 679 (2011).

[14] D. J. Korteweg and G. D. Vries, Philos. Mag. J. Sci. 39, $422(1895)$.

[15] E. Falcon, C. Laroche, and S. Fauve, Phys. Rev. Lett. 89, 204501 (2002). 